\title{
NQO1 potentiates apoptosis evasion and upregulates XIAP via inhibiting proteasome-mediated degradation SIRT6 in hepatocellular carcinoma
}

Hong-Zhong Zhou ${ }^{1+}$, Han-Qing Zeng ${ }^{2 \dagger}$, Ding Yuan ${ }^{3}$, Ji-Hua Ren ${ }^{1}$, Sheng-Tao Cheng ${ }^{1}$, Hai-Bo Yu ${ }^{1}$, Fang Ren ${ }^{1}$, Qing Wang ${ }^{1}$, Yi-Ping Qin ${ }^{1}$, Ai-Long Huang ${ }^{1}$ and Juan Chen ${ }^{1 *}$

\begin{abstract}
Background: Our previous study has demonstrated that $\mathrm{NAD}(\mathrm{P}) \mathrm{H}$ : quinone oxidoreductase 1 (NQO1) is significantly upregulated in human liver cancer where it potentiates the apoptosis evasion of liver cancer cell. However, the underlying mechanisms of the oncogenic function of NQO1 in HCC have not been fully elucidated.

Methods: Expression of NQO1, SIRT6, AKT and X-linked inhibitor of apoptosis protein (XIAP) protein were measured by western blotting and immunohistochemistry. Additionally, the interaction between NQO1 and potential proteins were determined by immunoprecipitation assays. Furthermore, the effect of NQO1 and SIRT6 on tumor growth was determined in cell model and orthotopic tumor implantation model.

Results: We found that NQO1 overexpression in HCC enhanced SIRT6 protein stability via inhibiting ubiquitinmediated 265 proteasome degradation. High level of SIRT6 reduced acetylation of AKT which resulted in increased phosphorylation and activity of AKT. Activated AKT subsequently phosphorylated anti-apoptotic protein XIAP at Ser87 which determined its protein stability. Reintroduction of SIRT6 or AKT efficiently rescued NQO1 knock-outmediated inhibition of growth and induction of apoptosis. In orthotopic mouse model, NQO1 knock-out inhibited tumor growth and induced apoptosis while this effect was effectively rescued by SIRT6 overexpression or MG132 treatment partially.
\end{abstract}

Conclusions: Collectively, these results reveal an oncogenic function of NQO1 in sustaining HCC cell proliferation through SIRT6/AKT/XIAP signaling pathway.

Keywords: NQO1, SIRT6, XIAP, Hepatocellular carcinoma, Apoptosis

\section{Background}

$\mathrm{NAD}(\mathrm{P}) \mathrm{H}$ : quinone oxidoreductase 1 (NQO1) is originally identified as an antioxidant enzyme which catalyzes the two-electron oxidoreduction to generate an unstable hydroquinone [1]. Recent studies have showed that NQO1 is upregulated in many human cancers, including

\footnotetext{
* Correspondence: chenjuan2014@cqmu.edu.cn

${ }^{\dagger}$ Hong-Zhong Zhou and Han-Qing Zeng contributed equally to this work. 'The Key Laboratory of Molecular Biology of Infectious Diseases designated by the Chinese Ministry of Education, Institute for Viral Hepatitis, Department of Infectious Diseases, The Second Affiliated Hospital, Chongqing Medical University, Room 617, College of Life Sciences Building, 1 YiXueYuan Road, YuZhong District, Chongqing 400016, China

Full list of author information is available at the end of the article
}

Cholangiocarcinoma [2], non-small cell lung cancer [3], uterine cervical cancer [4], prostate cancer [5, 6] and pancreatic cancer [7]. In breast, lung cancer and uterine cervical cancer, high-level NQO1 expression was closely associated with poor differentiation, late clinical stage and lymph node metastasis. Patients with high-level expression of NQO1 had a shorter disease-free survival and overall survival rate than those with low NQO1 expression in cell lung cancer [3]. Previously, we have reported that NQO1 is generally increased in HCCs where its high expression level is related with poor patient survival rate. NQO1 potentiates the apoptosis evasion of liver cancer cell through upregulating $\mathrm{X}$ -

(c) The Author(s). 2019 Open Access This article is distributed under the terms of the Creative Commons Attribution 4.0 International License (http://creativecommons.org/licenses/by/4.0/), which permits unrestricted use, distribution, and 
linked inhibitor of apoptosis protein (XIAP) protein stability [8]. However, the molecular mechanisms by which NQO1 exerts pro-tumorigenic function need to further elucidated.

Sirtuin 6 (SIRT6), a member of the sirtuin family, is $\mathrm{NAD}^{+}$-dependent deacetylases with important roles in glucose homeostasis, maintenance of genome stability and cancers development [9-11]. Because of its complex and opposite functional roles, SIRT6 is considered a two-edged sword in carcinogenesis [12]. In particular, SIRT6 deacetylates lysine 56 of histone H3 (H3K56) and lysine 9 of histone H3 (H3K9), which has a duty for reduced chromatin accessibility to transcription factors. Interestingly, it was found that acetylation of H3K56 is increased in multiple types of cancer including, liver, breast, thyroid and colon cancer [13, 14]. Importantly, SIRT6 plays a tumor suppressor role in the maintenance of cancer [15]. On the other hand, SIRT6 is overexpressed in some cancers, such as squamous cell carcinoma (SCC), breast cancer, ovarian cancer and multiple myeloma, suggesting it possesses oncogenic activity [16-19]. SIRT6 overexpression increases cancer cell proliferation by directly modulating oncogenic proteins or the acetylation of H3K9 and H3K56 of oncogene promoter dependent on its deacetylase activity $[13,14]$. In HCC, SIRT6 potentiates apoptosis evasion of $\mathrm{HCC}$ cells via chromatin remodeling. Mechanistically, SIRT6 induces H3K9 deacetylation that blocks Bax transcription, and enhances E2F-1 and p53 chromatin accessibility [20]. 3',5'-Cyclic adenosine monophosphate (cAMP), AKT, miR-34a and miR-122 are involved in the regulation of transactivation and expression of SIRT6 [16, 18, 21, 22]. However, the investigations on the stability of SIRT6 are limited.

Excepting its oxidoreductase activity, NQO1 has been reported to stabilize many proteins, including p33 [23], p53 [24], p73 [25], eIF4GI [26] and c-Fos [27], by inhibiting their proteasomal degradation. In this study, we demonstrated that NQO1 interacts physically with SIRT6, stabilizes the protein and prevents it from ubiquitindependent $26 \mathrm{~S}$ proteasomal degradation. Consequently, SIRT6 deacetylated AKT to promote its phosphorylation and activation, thus leading to increased XIAP phosphorylation and protein stability. Taken together, our results strongly suggested an important role of NQO1-induced SIRT6 stability in tumor biology of HCC.

\section{Methods}

\section{Cell lines}

Huh-7 and PLC/PRF/5 were obtained from the Heath Science Research Resource Bank (HSRRB) and American Type Culture Collection (ATCC), respectively. The two cell lines were routinely maintained in Dulbecco's modified Eagle's medium containing 10\% fetal bovine serum (Gibco-BRL) and penicillin/streptomycin, and cultured at $37^{\circ} \mathrm{C}$ with $5 \% \mathrm{CO}_{2}$ in a humidified incubator. Cell lines were authenticated by short tandem repeat analysis technology (STR), and examined negative for mycoplasma.

\section{Antibodies, plasmids and chemicals}

The pcDNA3-Xiap-Myc (\#11833) plasmid was purchased from Addgene (Cambridge, MA). The plasmids of Myc-DDK-tagged-SIRT6 (RC202833) and pCMV6NQO1 (SC119599) were obtained from OriGene Technologies (Rockville, MD). Rabbit anti-AKT (\#4691), rabbit anti-phospho-AKT (\#4060), mouse anti-ubiquitin (\#3936), rabbit anti-acetylated-Lysine (\#9441), rabbit anti-SIRT1 (\#9475), rabbit anti-SIRT2 (\#12650), rabbit anti-SIRT3 (\#2627), rabbit anti-SIRT5 (\#8782) and rabbit anti-Ki67 (\#9027) were bought from CST (Danvers, MA). Rabbit anti-SIRT6 (NB100-2522) was obtained from Novus Biologicals (Colorado, USA). Rabbit antiSIRT7 (S5947) was purchased from Sigma (Saint Louis, MO). Rabbit anti-26S (14748-1-AP) were obtained from Proteintech. Mouse anti-SIRT4 (SC-135798) was purchased from Santa Cruz. MG132 (S2619), MK-2206 2HCL (S1078) and PYR-41 (S7129) were obtained from Selleck Chemicals (Houston, USA). Cycloheximide (a protein synthesis inhibitor in eukaryotes [28]) was purchased from Calbiochem (Merck). Dicoumarol (the most potent inhibitor of NQO1 [29]) was purchased from MERCK (287897).

\section{Coimmunoprecipitation assay and western blotting analysis}

Coimmunoprecipitation assays were performed as described previously [20]. Briefly, cell lysates were precipitated in an immunoprecipitation assay buffer at $4{ }^{\circ} \mathrm{C}$ overnight with appropriated antibodies (anti-XIAP, antiSIRT6, anti-AKT, anti-NQO1, anti-26S). Proteins were separated by $8-12 \%$ SDS-PAGE, immunocomplexes then were subjected to western blotting analysis.

\section{Detection of proliferation and apoptotic cells}

To examine cell proliferation rate, the same number of cells were seeded into 6-well plates. Cell viability as well as cell number were assessed using trypan blue exclusion assay. Apoptotic process was detected according to the manufacturer's instructions. Then the cells were analyzed by flow cytometry immediately.

\section{Immunohistochemistry (IHC)}

Immunohistochemistry for target proteins were executed on paraffin sections using appreciated antibodies (anticleaved PARP, 1:100; anti-XIAP, 1:50; anti-NQO1,1:50; anti-SIRT6, 1:100). IHC was carried out as described by Sun et.al [30]. In brief, staining was assessed based on the percentage of positively stained cells. And the 
staining intensity was analyzed by Image-Pro Plus 6.0 software.

\section{Animal studies}

For tumor formation in nude mice, indicated cells $(1 \times$ $10^{6}$ ) were harvested in $30 \mu \mathrm{l}$ medium (DMEM: Matrigel $=1: 1)$ and were injected into the left lobe of liver of 5 or 6-week-old male nude mice. Thirty-two nude mice were divided into four groups randomly $(n=8)$. Nude mice received injections with or without MG132 (0.05 $\mathrm{mg} / \mathrm{kg}$, intraperitoneally, daily) for 30 days starting 5 days after the injection of indicated cells.

Histopathological examination was executed using IHC staining as previously described. All of the procedures for handling of animals abided by the guidelines of Chongqing Medical University Animal Care Committee (reference number: 2019002), and were approved by the institutional animal research ethical committee.

\section{Statistical analysis}

All statistical analyses were conducted using GraphPad Prism5 (GraphPad) software unless otherwise indicated. Data were shown as mean \pm S.E.M. from three independent experiments. For comparisons, a two tailed student's t test, Wilcoxon signed-rank test, Mann-Whitney test were performed as indicated. $p$ value $<0.05$ was considered significant statistically.

\section{Results}

NQ01 regulates XIAP phosphorylation via AKT activation As reported in our previous study [8], NQO1 was generally upregulated in HCC, which was associated with poor prognosis. NQO1 inhibitor or NQO1 knock-down/ knock-out reduced the level of XIAP protein via decreasing the phosphorylation status of XIAP at ser87. It has been reported that XIAP was phosphorylated and stabilized by AKT at Ser87 [31-34], we then analyzed whether NQO1 regulated AKT expression and activation. Interestingly, knock-down/knock-out of NQO1 reduced the phosphorylation status of AKT without affecting total expression of AKT, whereas NQO1 overexpression increased the level of phosphorylated AKT (Fig. 1a). Importantly, AKT inhibitor (MK2206) remarkably blocked NQO1-induced XIAP expression and phosphorylation (Fig. 1b). Introduction of AKT which increased the level of phosphorylated AKT markedly rescued NQO1 silencing-induced decrease of XIAP expression and phosphorylation (Fig. 1c). Moreover, introduction of AKT rescued NQO1 silencing-inducing growth inhibition and apoptosis in HCC cells (Fig. 1d-e). The data revealed NQO1 regulated the phosphorylation status of XIAP and the protein stability through AKT activation.

\section{SIRT6 involves in NQO1-mediated regulation of AKT signaling}

It has been reported that deacetylation of AKT by sirtuin family members, which are class III $\mathrm{NAD}^{+}$-dependent protein deacetylation, is necessary for its activation [35, 36]. On the other hand, NQO1 catalyzes the reduction of quinones to hydroquinone by utilizing $\mathrm{NADH}$ as an electron donor, which consequently increases intracellular $\mathrm{NAD}^{+}$levels [37]. Cellular $\mathrm{NAD}^{+}$acts as a cofactor for many enzymes, particularly the sirtuins [38]. These finding prompted us to hypothesize that NQO1-mediated regulation of sirtuin members might account for AKT phosphorylation and activation in HCC cells. To test the hypothesize, we first examined expression of sirtuin members (SIRT1-SIRT7) in NQO1-depleted HCC cells by realtime PCR and immunoblotting analysis. Knock-down of NQO1 markedly resulted in decreased protein level of SIRT6, whereas it had no obvious effect on other sirtuin members (Additional file 1: Figure S1a-b). The total protein levels of AKT and phosphorylated AKT were analyzed in sirtuin members (SIRT1-SIRT7) knock-down cells. Interestingly, SIRT1, SIRT2 and SIRT6 suppression decreased phosphorylation of AKT without affecting total AKT level (Additional file 2: Figure S2). To this end, we focus on SIRT6 for further investigation.

NQO1 increases XIAP phosphorylation via SIRT6/AKT axis

To identify whether XIAP is the downstream target of SIRT6, we first examined XIAP expression in SIRT6depleted cells. SIRT6 knock-down markedly decreased the level of both XIAP protein and phosphorylatedXIAP without affecting its mRNA level (Fig. 2a, and Additional file 3: Figure S3). To examine the role of SIRT6 on XIAP protein stability, SIRT6-depleted cells were treated with cycloheximide. When compared to control cells, the half-life of XIAP protein was obviously reduced in SIRT6-depleted cells (Fig. 2b). Under the treatment of MG132, the influence of SIRT6 depletion on XIAP was blocked which revealed SIRT6 can stabilize XIAP protein through inhibiting proteasomal degradation (Fig. 2c). XIAP has been shown to possess E3 ubiquitin ligase activity and phosphorylation of XIAP could promote its autoubiquitination. Thus, we next examine the effect of SIRT6 on XIAP ubiquitination. XIAP ubiquitination was markedly enhanced in cells co-expressing XIAP, shSIRT6 and HA-tagged ubiquitin (HA-Ub) compared to levels of control cells (Fig. 2d). Collectively, these results indicated that SIRT6 stabilizes XIAP protein from ubiquitin-mediated proteasome degradation.

Next, we further investigated the regulatory role of SIRT6 on AKT. SIRT6 knock-down significantly decreased phosphorylation of AKT, whereas it did not affect total protein of AKT (Fig. 3a). The coimmunoprecipitation assay confirmed the endogenous SIRT6 was coprecipitation with endogenous 
a

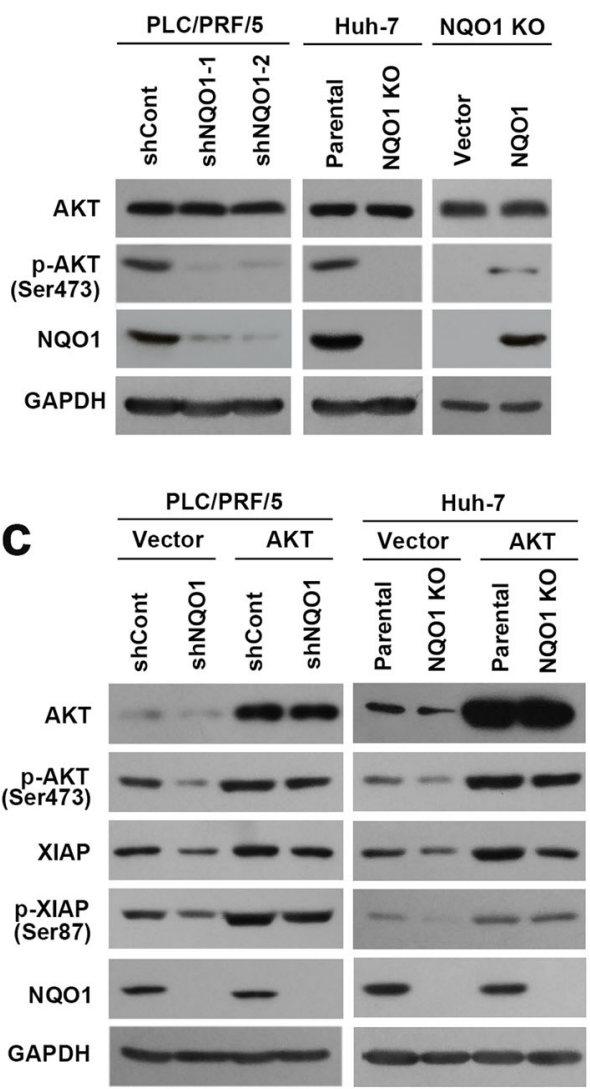

e

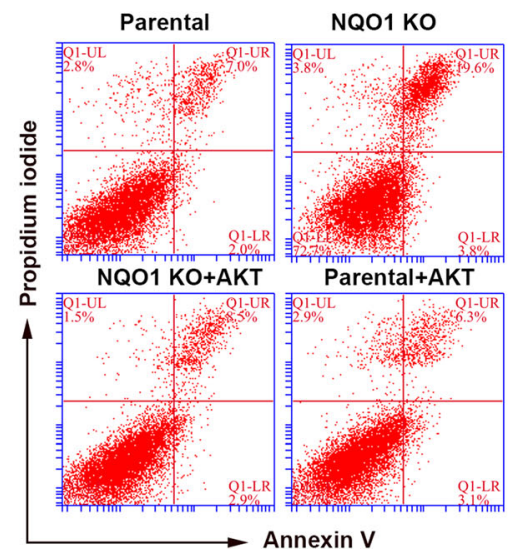

b

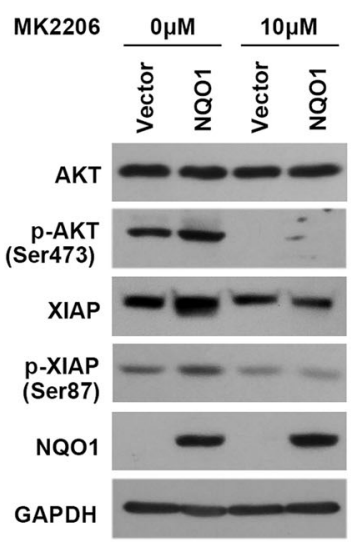

d
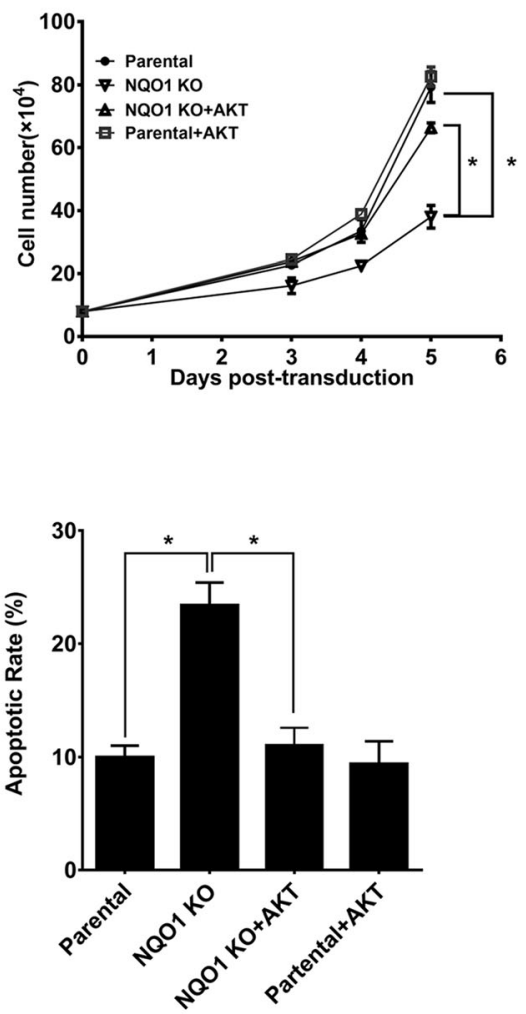

Fig. 1 NQO1 increases XIAP phosphorylation via AKT activation. a Immunoblotting analysis for AKT and phosphor-AKT (pSer473) in NQO1 knockdown/knock-out cells or NQO1 knock-out cells transfection of vector expressing NQO1. b Immunoblotting analysis for AKT, phosphor-AKT (pSer473), XIAP, and phosphor-XIAP (pSer87) in NQO1 knock-out cells. NQO1 cells were transfected with plasmid expressing NQO1 and then treated with AKT inhibitor MK2206 (10 $\mu \mathrm{M})$ for $24 \mathrm{~h}$. c Immunoblotting analysis for AKT, phosphor-AKT (pSer473), XIAP and phosphor-XIAP (pSer87) in NQO1 knock-down/knock-out cells transfected with AKT or empty vector. $\mathbf{d}$-e Trypan blue exclusion assay (d) and flow cytometry (e) were performed to analyze the NQO1-depleted cells transfected with AKT. Data are mean \pm SEM of $n=3$ independent experiments

AKT. This interaction was confirmed by ectopic expression of SIRT6 and AKT (Fig. 3b). SIRT6 silencing increased the acetylation level of AKT whereas SIRT6 overexpression exhibited opposite effect as determined by coimmunoprecipitation using anti-acetylation antibody (Fig. 3c). Moreover, immunoprecipitated AKT from HCC cells contained XIAP. Consistently, XIAP was coimmunoprecipitated with AKT (Fig. 3d). Importantly, AKT inhibitor (MK2206) remarkably inhibited SIRT6-induced increase of XIAP expression and phosphorylation (Fig. 3e). Introduction of AKT rescued 


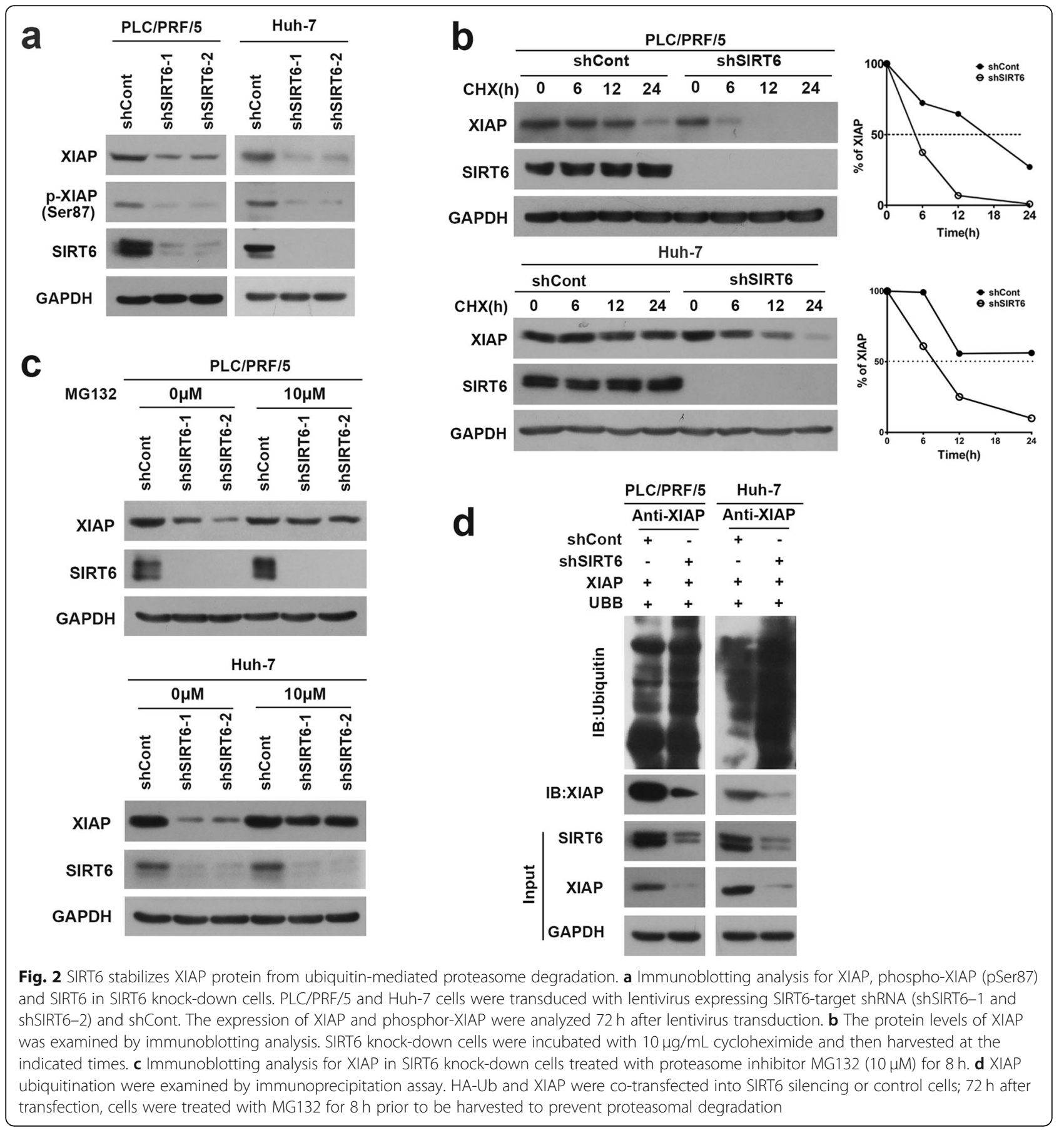

SIRT6-silencing-induced decrease of XIAP expression and phosphorylation (Fig. 3f). Collectively, these data demonstrated SIRT6 regulated phosphorylation and degradation of XIAP via deacetylation of AKT.

NQO1 stabilizes SIRT6 protein via blocking ubiquitinmediated proteasome degradation

To study the potential mechanism of NQO1 regulates SIRT6, we first analyzed the mRNA and protein level of SIRT6 in NQO1-depleted or dicoumarol-treated cells.
Consistently, loss-of-function of NQO1 (knock-down/ knock-out or chemical inhibitor) resulted in reduced SIRT6 protein without affecting its mRNA level (Fig. 4ac). To investigate whether NQO1 affected the stability of SIRT6 protein, we treated NQO1 knock-down cells with cycloheximide. When compared to control cells, the half-life of SIRT6 protein was obviously reduced in NQO1-depletion cells (Fig. 4d). The level of SIRT6 was restored in the presence of MG132 (Fig. 4e). These data supported the involvement of proteasomal machinery in 


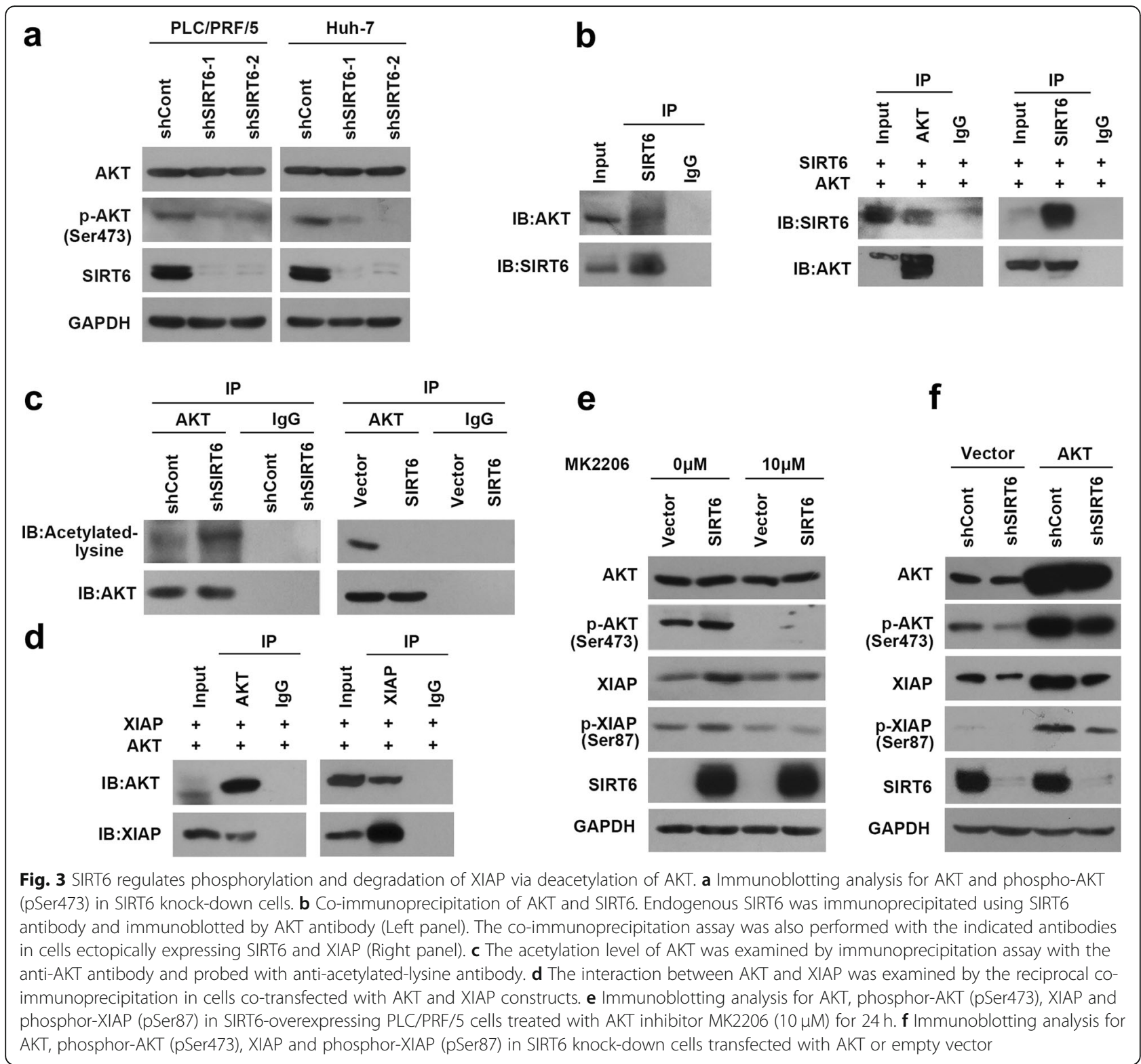

NQO1 depletion-mediated degradation of SIRT6. To assess whether NQO1 stabilized SIRT6 via inhibiting ubiquitination, HCC cells were treated with PYR-41 (50 $\mathrm{mM}$ ), a specific inhibitor of ubiquitin activating enzyme E1, thereby inhibiting whole-cell ubiquitination. PYR-41 treatment significantly restored the levels of SIRT6 protein in NQO1 depletion cells (Fig. 4f), suggesting that ubiquitination machinery was involved in NQO1 depletion-induced SIRT6 degradation. This was further confirmed by measuring the level of ubiquitinatedSIRT6 in NQO1 knock-down/knock-out cells. Lossof-function of NQO1 increased the level of ubiquitination in SIRT6 (Fig. 4g).

It has been reported that NQO1 stabilizes numerous proteins by physically binding to them and inhibiting ubiquitination [28, 39]. Therefore, we further identified whether NQO1 was physically related with SIRT6. Reciprocal co-immunoprecipitation assay with anti-NQO1 or anti-SIRT6 antibodies revealed the interaction between NQO1 and SIRT6 (Fig. 5a-b). On the other hand, NQO1 has been reported to regulate proteasomal degradation in a NADH-dependent manner [40]. Concordantly, we found that SIRT6 expression was remarkably increased in the presence of NQO1 cofactors NADH (Fig. 5c). Remarkable increase in the binding of NQO1 to SIRT6 was observed in the presence of $\mathrm{NADH}$, whereas dicoumarol that competing with NADH for binding to NQO1 decrease the association between NQO1 and SIRT6 (Fig. 5d-e). These data indicated that NQO1 interacts physically with SIRT6 which requires binding to NADH. 


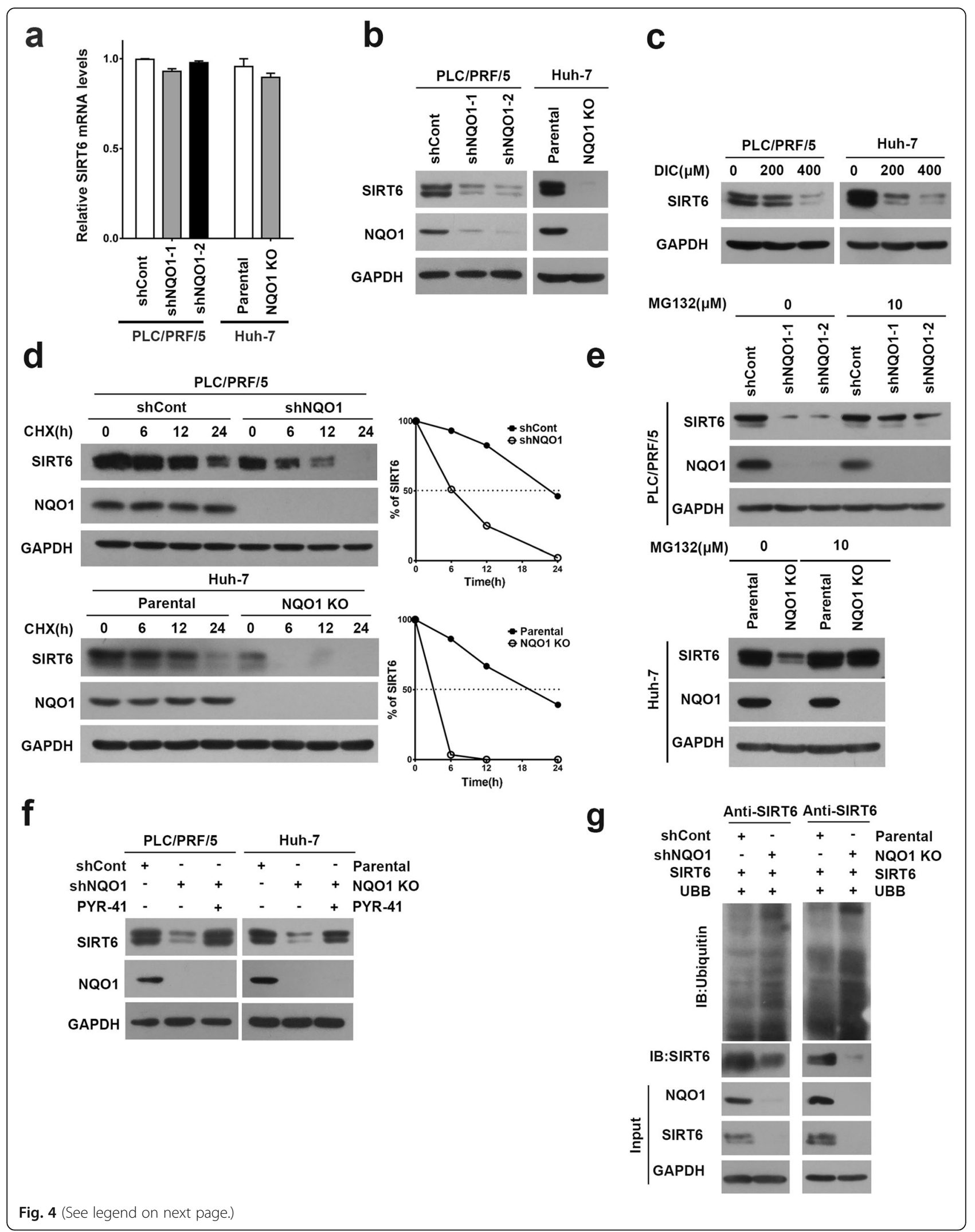


(See figure on previous page.)

Fig. 4 NQO1 knock-down/knock-out decreases the protein stability of SIRT6. a Real-time PCR for SIRT6 mRNA level in NQO1 knock-down PLC/ PRF/5 and NQO1 knock-out Huh-7 cells. Data are mean \pm SEM of $n=3$ independent experiments. ${ }^{*} p<0.05$. $\mathbf{b}$ Immunoblotting analysis for SIRT6 in NQO1 knock-down/knock-out cells. c Immunoblotting analysis for SIRT6 in PLC/PRF/5 and Huh-7 cells treated with dicoumarol (200 $\mu$ M or $400 \mu \mathrm{M}$ ) for $4 \mathrm{~h}$. $\mathbf{d}$ Immunoblotting analysis for SIRT6 in NQO1 knock-down/knock-out cells treated with $10 \mu \mathrm{g} / \mathrm{ml}$ cycloheximide at the indicated

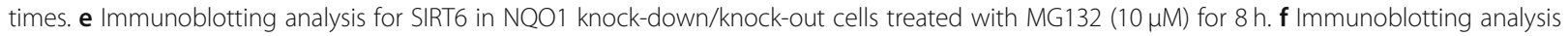
of SIRT6 in NQO1-knock-down/knock-out cells treated with PYR-41 (50 $\mu \mathrm{M})$ for $6 \mathrm{~h}$. g SIRT6 ubiquitination was examined by immunoprecipitation assay in PLC/PRF/5 and Huh-7 cells

Moreover, reciprocal co-immunoprecipitation assay revealed that NQO1 and SIRT6 were physically associated with $26 \mathrm{~S}$ proteasome in HCC cells (Fig. $5 \mathrm{f}$ ). These results indicated that NQO1 prevented SIRT6 from ubiquitinmediated proteasome degradation. Furthermore, introduction of SIRT6 restored XIAP protein and rescued NQO1 depletion-inducing growth inhibition and apoptosis in HCC cells (Additional file 4: Figure S4). Collectively, these results demonstrated that NQO1 exerts its oncogenic function through stabilizing SIRT6, which consequently leads to AKT activation and XIAP phosphorylation.

\section{Reintroduction of SIRT6 rescues NQO1 depletion-induced apoptosis in mouse model}

To study the oncogenic role of NQO1 in vivo, NQO1 knock-out cells with or without stable expression of

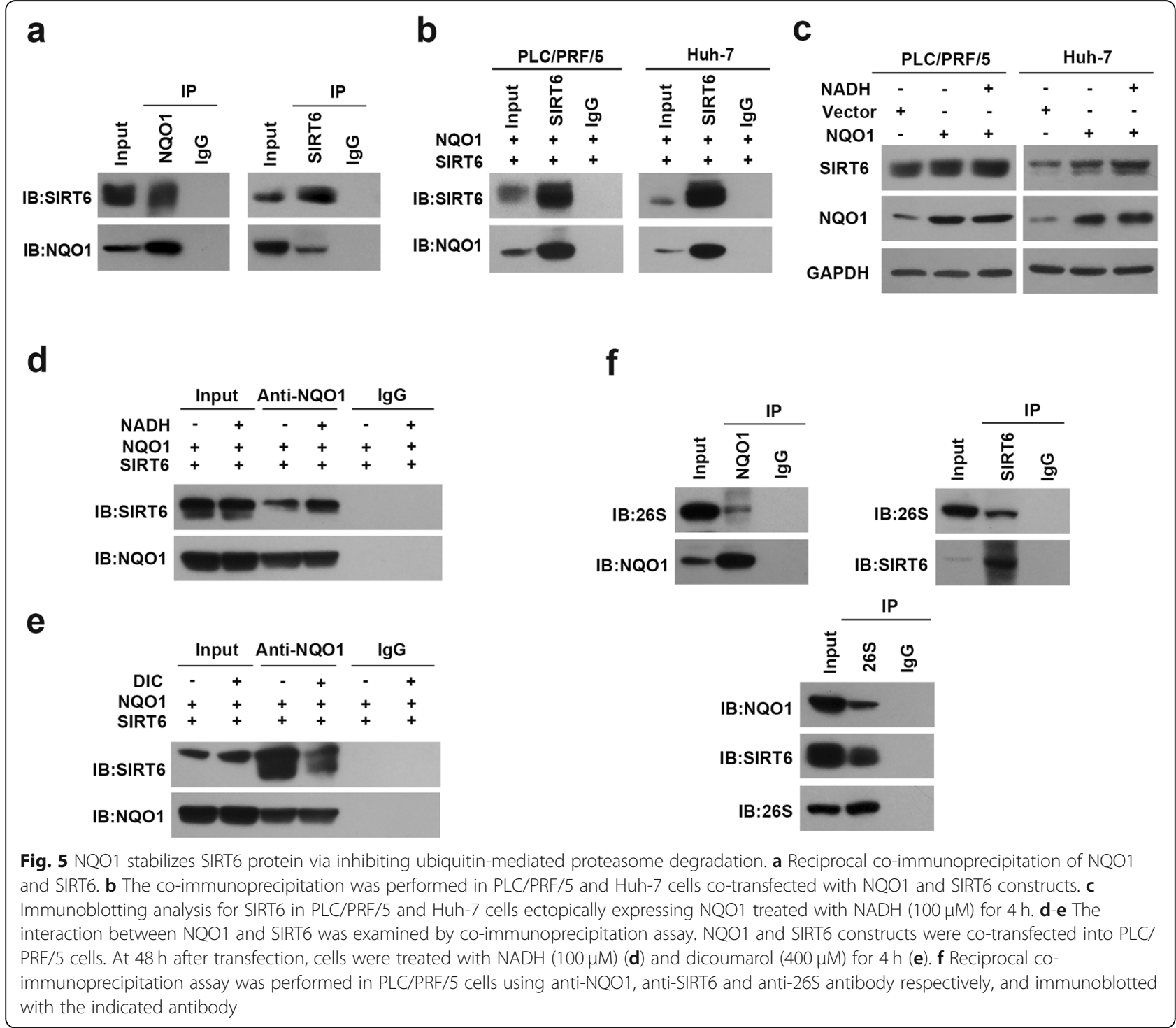


SIRT6 or parental cell were orthotopically injected into livers. When compared to the parental cells, we verified that NQO1 knock-out significantly suppressed the HCC tumor sizes in orthotopically transplanted HCC mice.

Meanwhile, SIRT6 overexpression rescued the effect of NQO1 knock-out-mediated growth inhibitory (Fig. 6a). To investigate whether ubiquitin-proteasome pathway was involved in NQO1 depletion-induced apoptosis in vivo, NQO1 knock out groups were received injections with or without MG132. As shown in Fig. 6a, MG132 treatment blocked tumor growth inhibition induced by NQO1 knock out. Furthermore, IHC analysis showed that NQO1 knock-out was related with reduced level of SIRT6, XIAP and increased level of cleaved
PARP in tumor tissues. Importantly, the above effects could be reversed by SIRT6 overexpression or MG132 treatment partially (Fig. 6b). These data suggested that MG132 attenuates NQO1 knock out-induced downregulation of SIRT6 and activation of SIRT6-XIAP axis.

\section{Discussion}

As shown in previous studies, NQO1 overexpression in HCC cells enhanced XIAP protein stability through promoting its phosphorylation [8], which determined its activity of autoubiquitination/ubiquitination and protein stability $[41,42]$. In this manuscript, we investigated the underlying mechanism how NQO1 regulated XIAP phosphorylation. It has been reported that the

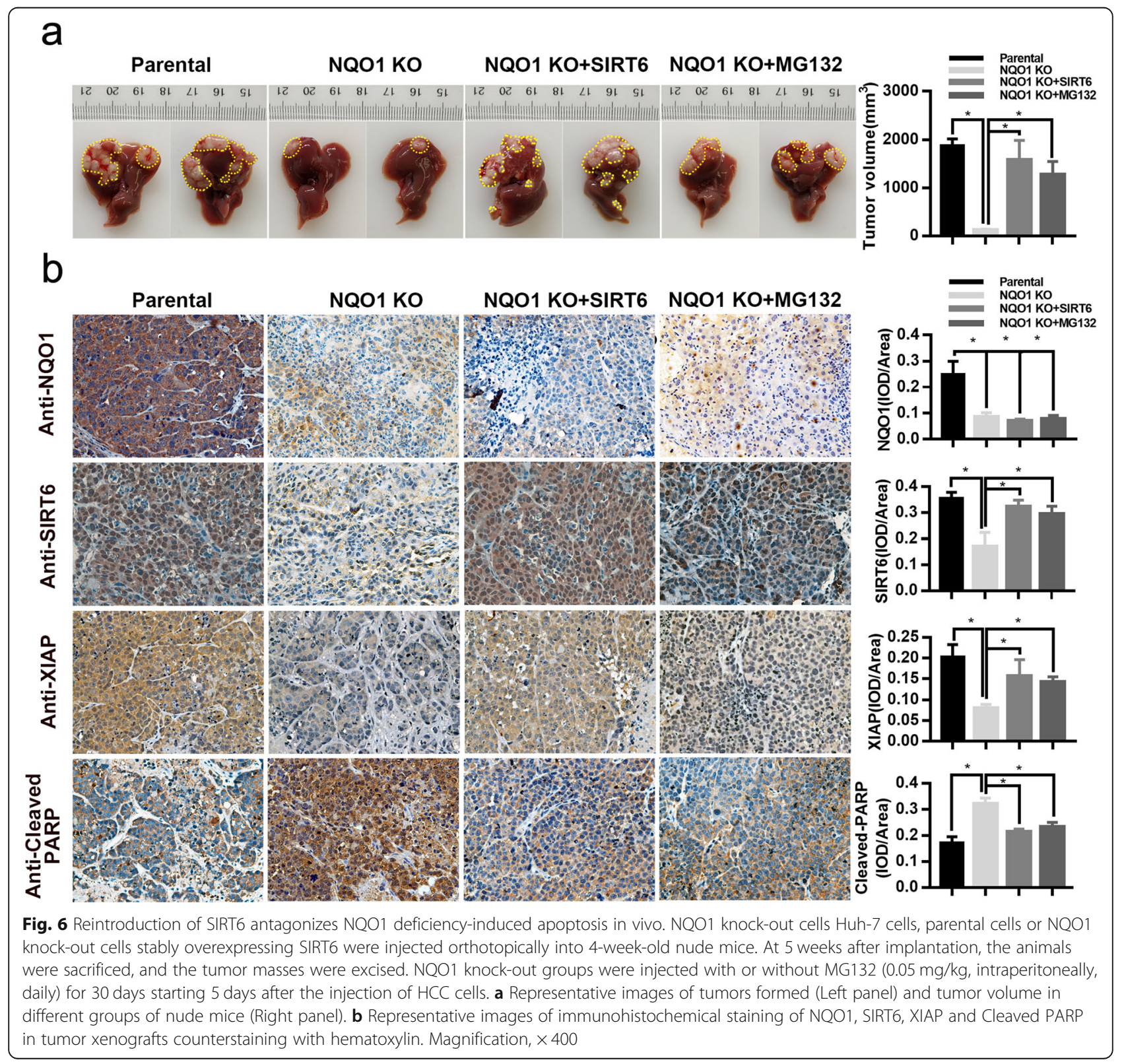


phosphorylation status at Serine 87 of XIAP could be regulated by AKT protecting it from ubiquitination and degradation [34, 41], which prompting us to hypothesize that in our model XIAP may be a downstream target of AKT. Consistent with previous studies, our data suggested loss-of-function of NQO1 reduced the phosphorylated status of AKT without affecting total AKT expression, whereas NQO1 overexpression increased level of phosphorylated AKT. Importantly, AKT inhibitor (MK2206) remarkably blocked NQO1-induced increase of XIAP protein and phosphorylation level, whereas increased phosphorylated AKT restored XIAP protein and phosphorylation level in NQO1-deleted cells. These data suggested NQO1 could affect AKT phosphorylation and activation to promote XIAP phosphorylation and stability in HCC cells.

Acetylation is one of mechanisms participated in the direct or indirect regulation of AKT activation [35, 43, 44]. Earlier studies have reported that deacetylation of AKT by SIRT1, SIRT2 and SIRT7, which are class III NAD ${ }^{+}$ dependent protein deacetylases, is necessary for its activation [35, 36]. SIRT1 deacetylated the two conserved lysine residues of AKT PH domain, contributing to the binding of AKT to PIP3, and thereby increasing membrane localization and activation [35]. Our previous study has revealed SIRT2 regulated the deacetylation and activation of AKT, which subsequently activated GSK3 $\beta / \beta$-catenin-signaling axis to regulate EMT in HCC [36]. Additionally, SIRT7 was also reported to regulate FKBP51 acetylation and enhances complex formation of FKBP51-AKT-PHLPP. This subsequently suppressed AKT pathway and then increased chemo-sensitivity in breast cancer cell [43]. Consistently, our present data demonstrated SIRT6 as downstream protein of NQO1 which was responsible for AKT activation by screening sirtuin members. Mechanistically, SIRT6 deacetylated and activated AKT, thus leading to increased XIAP phosphorylation and protein stability in HCC cells. However, our finding was inconsistent with previously published results that SIRT6 overexpression negatively regulated PI3K signaling pathway without affecting AKT phosphorylation. It was on account of SIRT6 exerted anti-tumor sphere-forming at the transcriptional level which was independent of SIRT6 histone deacetylase activity [45]. The contradictory of different function of SIRT6 in these studies may be result from tissue-specific molecular profiles, the context and gene dose.

NQO1 exercises a selective "gatekeeping" role in regulating the proteasomal degradation of specific proteins. NQO1 binds to and thereby stabilizes the important tumor suppressor p53 against $20 \mathrm{~S}$ proteasomal degradation [46]. In addition to p53, NQO1 also regulates the ubiquitin-independent $20 \mathrm{~S}$ proteasomal degradation of p73 $\alpha$ and p33 [23, 40]. Downregulation of NQO1 by HIV Rev. induces ubiquitin-independent proteasomal degradation of Tat, which is implicated in HIV-gene expression and latency [47]. But recently, Oh et.al has revealed that NQO1 stabilizes HIF-1 $\alpha$ by inhibiting the level of ubiquitination and the $26 \mathrm{~S}$ proteasomal

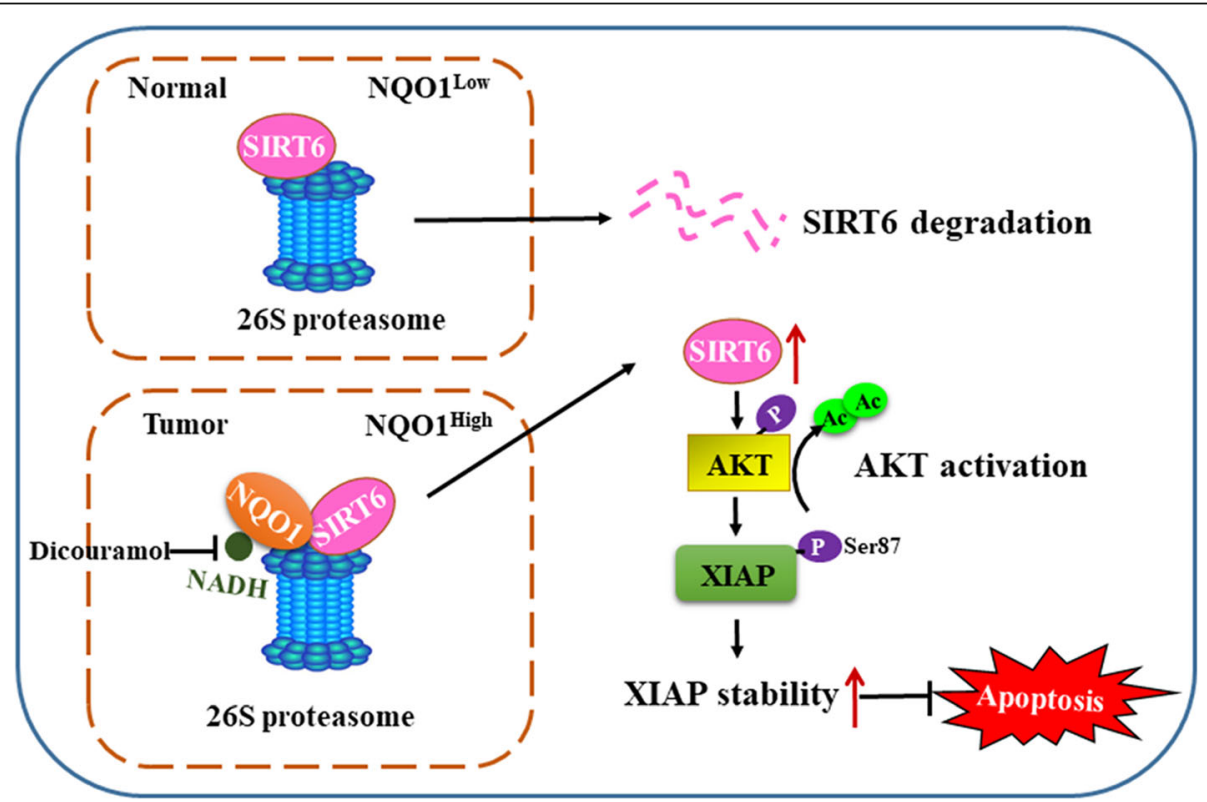

Fig. 7 Schematic model of how NQO1 inhibits HCC apoptosis. The working model for oncogenic role of NQO1 in HCC. In HCC cells where NQO1 expression is high, NQO1 interacts physically with SIRT6, stabilizes the protein and prevents it from ubiquitin-dependent proteasomal degradation. Consequently, SIRT6 deacetylated AKT to promote its phosphorylation and activation, thus leading to increasing XIAP phosphorylation and protein stability 
degradation [28]. Consistent with Oh et.al, we found that both NQO1 and SIRT6 were physically associated with $26 \mathrm{~S}$ proteasomes in HCC cells, suggesting that NQO1 stabilizes SIRT6 by blocking ubiquitinationdependent proteasomal degradation. This finding was further confirmed in vivo. MG132 treatment blocked tumor growth inhibition induced by NQO1 knock out, accompanied with increased level of SIRT6 and XIAP. MG132, which acts as a blocker in ubiquitin-proteasome pathway, is involved in $>80 \%$ of intracellular protein degradation. However, its role in apoptosis of cancer cell is controversial. MG132 promotes the cisplatin-induced apoptosis and inhibits tumor growth [48, 49], however, it blocks high-dose UV irradiation-induced apoptosis [50]. Additionally, MG132 also blocks bufalin-induced cell apoptosis by preventing the degradation of antiapoptotic Bcl-2 family member (Mcl-1) [51]. In this study, MG132 treatment blocks NQO1 depletioninduced apoptosis, supporting its role in inhibiting apoptosis. Basing on our current data, we suggest that NQO1 binds to SIRT6 and inhibits ubiquitin-dependent proteasomal degradation.

\section{Conclusions}

In summary, our findings determined NQO1 exerts its oncogenic function by regulating SIRT6/AKT/XIAP pathway. In HCC cells where NQO1 expression is high, NQO1 physically interacts with SIRT6 and stabilizes its protein against ubiquitin-dependent proteasomal degradation. Consequently, SIRT6 deacetylated AKT to promote its phosphorylation and activation, thus leading to increase XIAP phosphorylation and protein stability (Fig. 7). Our findings provide insights on the meaning of SIRT6/ AKT/XIAP axis for NQO1-mediated tumorigenesis.

\section{Supplementary information}

Supplementary information accompanies this paper at https://doi.org/10. 1186/s12964-019-0491-7.

Additional file 1: Figure S1. Effect of NQO1 silencing on seven sirtuin members. (a) Real-time PCR for SIRT1-7 mRNA level in NQO1 knockdown PLC/PRF/5 cells. Data are mean \pm SEM of $n=3$ independent experiments. (b) Immunoblotting analysis for SIRT1-7 in NQO1-silencing cells.

Additional file 2: Figure S2. Effect of sirtuin family members silencing on AKT. Immunoblotting analysis for total AKT and phospho-AKT in sirtuin members (SIRT1-SIRT7) knock-down cells.

Additional file 3: Figure S3. Effect of SIRT6 silencing on XIAP. Realtime PCR for XIAP mRNA level in SIRT6 knock-down PLC/PRF/5 and Huh7 cells. Data are mean \pm SEM of $n=3$ independent experiments.

Additional file 4: Figure S4. Ectopic expression SIRT6 antagonizes the effect of NQO1 deficiency on proliferation and apoptosis. (a) Immunoblotting analysis for NQO1, SIRT6, XIAP and Cleaved-PARP in NQO1-depleted PLC/PRF/5 cells transfected with vector expressing SIRT6. (b) Trypan blue exclusion assay for NQO1-depleted PLC/PRF/5 cells or control cells transfected with vector expressing SIRT6. (c) Flow cytometry with Annexin V/Pi for NOO1-depleted PLC/PRF/5 cells transfected with vector expressing SIRT6.

\section{Abbreviations}

CHX: Cycloheximide; DIC: Dicoumarol; HCC: Hepatocellular Carcinoma; NAD: Nicotinamide adenine dinucleotide; NADH: Nicotinamide adenine dinucleotide plus hydrogen; NADPH: Nicotinamide adenine dinucleotide phosphate plus hydrogen; NQO1: NAD(P)H: quinone oxidoreductase 1; SIRT6: Sirtuin 6; UBB: Ubiquitin B gene; XIAP: X-linked inhibitor of apoptosis protein

\section{Acknowledgements}

We would like to thank the First Affiliated Hospital of Chongqing Medical University which provide the primary HCC samples and corresponding adjacent nontumorous (NT) liver specimens. We appreciate the service of the Animal Center of Chongqing Medical University.

\section{Authors' contributions}

JC designed the experiments. H-ZZ, J-HR, S-TC, H-BY, FR, QW and Y-PQ performed the experiments. H-QZ, DY, and A-LH provided clinical specimens and mouse model. $\mathrm{H}-\mathrm{ZZ}, \mathrm{H}-\mathrm{QZ}$ and DY analyzed the data. JC and $\mathrm{H}-\mathrm{ZZ}$ wrote the manuscript. A-LH and JC. supervised the project and held with the writing of the manuscript. All authors discussed the results and commented on manuscript. All authors read and approved the final manuscript.

\section{Funding}

The work was supported by National Natural Science Foundation of China (Grant No. 81871656, 81802437, 8181101099 and 81922011), Creative Research Group of CQ University (CXQT19016), Chongqing Natural Science Foundation (cstc2018jcyjAX0114) and National Science and Technology Major Project (2017ZX10202203).

Availability of data and materials

All data used in this study are fully available without restrictions.

\section{Ethics approval and consent to participate}

This study was approved by Ethics Committee of Chongqing Medical University, and all patients were provided informed consent. All of the procedures for handling of animals abided by the guidelines of Chongqing medical University Animal Care Committee, and were approved by the institutional animal research ethical committee.

\section{Consent for publication}

All contributing authors agree to the publication of this article.

\section{Competing interests}

The authors declare that they have no competing interests.

\section{Author details}

${ }^{1}$ The Key Laboratory of Molecular Biology of Infectious Diseases designated by the Chinese Ministry of Education, Institute for Viral Hepatitis, Department of Infectious Diseases, The Second Affiliated Hospital, Chongqing Medical University, Room 617, College of Life Sciences Building, 1 YiXueYuan Road, YuZhong District, Chongqing 400016, China. ' ${ }^{2}$ Department of Hematology, The Second Affiliated Hospital, Chongqing Medical University, Chongqing, China. ${ }^{3}$ Department of Neurosurgery, The Fifth People's Hospital of Chongqing, Chongqing, China.

Received: 15 July 2019 Accepted: 26 November 2019 Published online: 16 December 2019

\section{References}

1. Huang X, Motea EA, Moore ZR, Yao J, Dong Y, Chakrabarti G, Kilgore JA, Silvers MA, Patidar PL, Cholka A, et al. Leveraging an NQO1 Bioactivatable Drug for Tumor-Selective Use of Poly(ADP-ribose) Polymerase Inhibitors. Cancer Cell. 2016;30:940-52.

2. Zeekpudsa P, Kukongviriyapan V, Senggunprai L, Sripa B, Prawan A. Suppression of $\mathrm{NAD}(\mathrm{P}) \mathrm{H}$-quinone oxidoreductase 1 enhanced the susceptibility of cholangiocarcinoma cells to chemotherapeutic agents. J Exp Clin Cancer Res. 2014;33:11. 
3. Li Z, Zhang Y, Jin T, Men J, Lin Z, Qi P, Piao Y, Yan G. NQO1 protein expression predicts poor prognosis of non-small cell lung cancers. BMC Cancer. 2015;15:207.

4. Ma Y, Kong J, Yan G, Ren X, Jin D, Jin T, Lin L, Lin Z. NQO1 overexpression is associated with poor prognosis in squamous cell carcinoma of the uterine cervix. BMC Cancer. 2014;14:414.

5. Thapa D, Meng P, Bedolla RG, Reddick RL, Kumar AP, Ghosh R. NQO1 suppresses NF-kappaB-p300 interaction to regulate inflammatory mediators associated with prostate tumorigenesis. Cancer Res. 2014;74:5644-55.

6. Kurfurstova D, Bartkova J, Vrtel R, Mickova A, Burdova A, Majera D, Mistrik M, Kral M, Santer FR, Bouchal J, Bartek J. DNA damage signalling barrier, oxidative stress and treatment-relevant DNA repair factor alterations during progression of human prostate cancer. Mol Oncol. 2016;10:879-94.

7. Silvers MA, Deja S, Singh N, Egnatchik RA, Sudderth J, Luo X, Beg MS, Burgess SC, DeBerardinis RJ, Boothman DA, Merritt ME. The NQO1 bioactivatable drug, beta-lapachone, alters the redox state of NQO1+ pancreatic cancer cells, causing perturbation in central carbon metabolism. J Biol Chem. 2017;292:18203-16.

8. Li W-Y, Zhou H-Z, Chen Y, Cai X-F, Tang H, Ren J-H, Wai Wong VK, Kwan Law BY, Chen Y, Cheng S-T, et al. NAD(P)H: quinone oxidoreductase 1 overexpression in Hepatocellular Carcinoma potentiates apoptosis evasion through regulating stabilization of $X$-linked inhibitor of apoptosis protein. Cancer Letters. 2019.

9. Zhang W, Wan H, Feng G, Qu J, Wang J, Jing Y, Ren R, Liu Z, Zhang L, Chen $Z$, et al. SIRT6 deficiency results in developmental retardation in cynomolgus monkeys. Nature. 2018;560:661-5.

10. Naiman S, Cohen HY. Role for the longevity protein SIRT6 in primate development. Nature. 2018;560:559-60.

11. Vitiello M, Zullo A, Servillo L, Mancini FP, Borriello A, Giovane A, Della Ragione F, D'Onofrio N, Balestrieri ML. Multiple pathways of SIRT6 at the crossroads in the control of longevity, cancer, and cardiovascular diseases. Ageing Res Rev. 2017:35:301-11.

12. Desantis V, Lamanuzzi A, Vacca A. The role of SIRT6 in tumors. Haematologica. 2018;103:1-4

13. Michishita E, McCord RA, Berber E, Kioi M, Padilla-Nash H, Damian M, Cheung P, Kusumoto R, Kawahara TLA, Barrett JC, et al. SIRT6 is a histone H3 lysine 9 deacetylase that modulates telomeric chromatin. Nature. 2008;452:492-6.

14. Kawahara TLA, Michishita E, Adler AS, Damian M, Berber E, Lin M, McCord RA, Ongaigui KCL, Boxer LD, Chang HY, Chua KF. SIRT6 Links Histone H3 Lysine 9 Deacetylation to NF-KB-Dependent Gene Expression and Organismal Life Span. Cell. 2009;136:62-74.

15. Lerrer B, Gertler AA, Cohen HY. The complex role of SIRT6 in carcinogenesis. Carcinogenesis. 2016;37:108-18.

16. Ming M, Han W, Zhao B, Sundaresan NR, Deng C-X, Gupta MP, He Y-Y. SIRT6 Promotes COX-2 Expression and Acts as an Oncogene in Skin Cancer. Cancer Res. 2014;74:5925-33.

17. Gomes AR, Koo C-Y, Yagüe E, Monteiro LJ, Khongkow M, Harada-Shoji N, Khongkow P, Coombes RC, Laohasinnarong S, Cavaco TB, et al. SIRT6 modulates paclitaxel and epirubicin resistance and survival in breast cancer. Carcinogenesis. 2013;34:1476-86.

18. Elhanati S, Ben-Hamo R, Kanfi Y, Varvak A, Glazz R, Lerrer B, Efroni S, Cohen HY. Reciprocal Regulation between SIRT6 and miR-122 Controls Liver Metabolism and Predicts Hepatocarcinoma Prognosis. Cell Rep. 2016;14: 234-42.

19. Cea M, Cagnetta A, Adamia S, Acharya C, Tai Y-T, Fulciniti M, Ohguchi H, Munshi A, Acharya P, Bhasin MK, et al. Evidence for a role of the histone deacetylase SIRT6 in DNA damage response of multiple myeloma cells. Blood. 2016;127:1138-50

20. Ran L-K, Chen Y, Zhang Z-Z, Tao N-N, Ren J-H, Zhou L, Tang H, Chen X, Chen K, Li W-Y, et al. SIRT6 Overexpression Potentiates Apoptosis Evasion in Hepatocellular Carcinoma via BCL2-Associated X Protein-Dependent Apoptotic Pathway. Clin Cancer Res. 2016;22:3372-82.

21. Lefort K, Brooks Y, Ostano P, Cario-André M, Calpini V, Guinea-Viniegra J, Albinger-Hegyi A, Hoetzenecker W, Kolfschoten I, Wagner EF, et al. A miR34a-SIRT6 axis in the squamous cell differentiation network. EMBO J. 2013; 32:2248-63.

22. Kim E-J, Juhnn Y-S. Cyclic AMP signaling reduces sirtuin 6 expression in non-small cell lung cancer cells by promoting ubiquitin-proteasomal degradation via inhibition of the Raf-MEK-ERK (Raf/mitogen-activated extracellular signal-regulated kinase/extracellular signal-regulated kinase) pathway. J Biol Chem. 2015;290:9604-13.
23. Garate M, Wong RPC, Campos El, Wang Y, Li G. NAD(P)H quinone oxidoreductase 1 inhibits the proteasomal degradation of the tumour suppressor p33(ING1 b). EMBO Rep. 2008;9:576-81.

24. Gong X, Kole L, Iskander K, Jaiswal AK. NRH:Quinone Oxidoreductase 2 and NAD(P)H:Quinone Oxidoreductase 1 Protect Tumor Suppressor p53 against $20 S$ Proteasomal Degradation Leading to Stabilization and Activation of p53. Cancer Res. 2007;67:5380-8.

25. Levy D, Adamovich Y, Reuven N, Shaul Y. The Yes-associated protein 1 stabilizes p73 by preventing Itch-mediated ubiquitination of p73. Cell Death Differ. 2006;14:743.

26. Alard A, Fabre B, Anesia R, Marboeuf C, Pierre P, Susini C, Bousquet C, Pyronnet S. NAD(P)H Quinone-Oxydoreductase 1 Protects Eukaryotic Translation Initiation Factor 4GI from Degradation by the Proteasome. Mol Cell Biol. 2010;30:1097-105.

27. Adler J, Reuven N, Kahana C, Shaul Y. c-Fos Proteasomal Degradation Is Activated by a Default Mechanism, and Its Regulation by NAD(P)H:Quinone Oxidoreductase 1 Determines c-Fos Serum Response Kinetics. Mol Cell Biol. 2010;30:3767-78.

28. Oh ET, Kim JW, Kim JM, Kim SJ, Lee JS, Hong SS, Goodwin J, Ruthenborg RJ, Jung MG, Lee HJ, et al. NQO1 inhibits proteasome-mediated degradation of HIF-1alpha. Nat Commun. 2016;7:13593.

29. Chen M, Gong L, Qi X, Xing G, Luan Y, Wu Y, Xiao Y, Yao J, Li Y, Xue X, et al. Inhibition of renal NQO1 activity by dicoumarol suppresses nitroreduction of aristolochic acid I and attenuates its nephrotoxicity. Toxicol Sci. 2011;122:288-96.

30. Sun H, Xu J, Huang Q, Huang M, Li K, Qu K, Wen H, Lin R, Zheng M, Wei H, et al. Reduced CD160 Expression Contributes to Impaired NK-cell Function and Poor Clinical Outcomes in Patients with HCC. Cancer Res. 2018;78: 6581-93.

31. Ng VH, Hang BI, Sawyer LM, Neitzel LR, Crispi EE, Rose KL, Popay TM, Zhong A, Lee LA, Tansey WP, et al. Phosphorylation of XIAP at threonine 180 controls its activity in Wnt signaling. J Cell Sci. 2018;131:jcs210575.

32. Hou Y, Allan LA, Clarke PR. Phosphorylation of XIAP by CDK1-cyclin-B1 controls mitotic cell death. J Cell Sci. 2017;130:502-11.

33. Durand JK, Zhang Q, Baldwin AS. Roles for the IKK-Related Kinases TBK1 and IKKepsilon in Cancer. Cells. 2018;7:139.

34. Castells M, Milhas D, Gandy C, Thibault B, Rafii A, Delord JP, Couderc B. Microenvironment mesenchymal cells protect ovarian cancer cell lines from apoptosis by inhibiting XIAP inactivation. Cell Death Dis. 2013;4:e887.

35. Sundaresan NR, Pillai VB, Wolfgeher D, Samant S, Vasudevan P, Parekh V, Raghuraman H, Cunningham JM, Gupta M, Gupta MP. The deacetylase SIRT1 promotes membrane localization and activation of Akt and PDK1 during tumorigenesis and cardiac hypertrophy. Sci Signal. 2011;4:ra46.

36. Chen J, Chan AW, To KF, Chen W, Zhang Z, Ren J, Song C, Cheung YS, Lai $\mathrm{PB}$, Cheng SH, et al. SIRT2 overexpression in hepatocellular carcinoma mediates epithelial to mesenchymal transition by protein kinase B/glycogen synthase kinase-3beta/beta-catenin signaling. Hepatology. 2013;57:2287-98.

37. Siegel D, Dehn DD, Bokatzian SS, Quinn K, Backos DS, Di Francesco A, Bernier M, Reisdorph N, de Cabo R, Ross D. Redox modulation of NQO1. PLoS One. 2018:13:e0190717.

38. Canto C, Auwerx J. NAD+ as a signaling molecule modulating metabolism. Cold Spring Harb Symp Quant Biol. 2011;76:291-8.

39. Oh ET, Park HJ. Implications of NQO1 in cancer therapy. BMB Rep. 2015;48: 609-17.

40. Asher G, Tsvetkov P, Kahana C, Shaul Y. A mechanism of ubiquitinindependent proteasomal degradation of the tumor suppressors p53 and p73. Genes Dev. 2005;19:316-21.

41. Kim S, Lee TJ, Park JW, Kwon TK. Overexpression of CFLIPs inhibits oxaliplatin-mediated apoptosis through enhanced XIAP stability and Akt activation in human renal cancer cells. J Cell Biochem. 2008;105:971-9.

42. Kato K, Tanaka T, Sadik G, Baba M, Maruyama D, Yanagida K, Kodama T, Morihara T, Tagami S, Okochi M, et al. Protein kinase C stabilizes X-linked inhibitor of apoptosis protein (XIAP) through phosphorylation at Ser(87) to suppress apoptotic cell death. Psychogeriatrics. 2011;11:90-7.

43. Yu J, Qin B, Wu F, Qin S, Nowsheen S, Shan S, Zayas J, Pei H, Lou Z, Wang L. Regulation of Serine-Threonine Kinase Akt Activation by NAD $<$ sup $>+$ </sup>-Dependent Deacetylase SIRT7. Cell Rep. 2017;18:1229-40.

44. Srivastava S, Mohibi S, Mirza S, Band H, Band V. Epidermal Growth Factor Receptor activation promotes ADA3 acetylation through the AKT-p300 pathway. Cell Cycle. 2017;16:1515-25.

45. Ioris RM, Galié M, Ramadori G, Anderson JG, Charollais A, Konstantinidou G, Brenachot X, Aras E, Goga A, Ceglia N, et al. SIRT6 Suppresses Cancer Stem- 
like Capacity in Tumors with PI3K Activation Independently of Its Deacetylase Activity. Cell Rep. 2017;18:1858-68.

46. Anwar A, Dehn D, Siegel D, Kepa JK, Tang L, Pietenpol JA, Ross D. Interaction of human $\mathrm{NAD}(\mathrm{P}) \mathrm{H}$ :quinone oxidoreductase 1 (NQO1) with the tumor suppressor protein p53 in cells and cell-free systems. J Biol Chem. 2003;278:10368-73.

47. Lata S, Ali A, Sood V, Raja R, Banerjea AC. HIV-1 Rev downregulates Tat expression and viral replication via modulation of $\mathrm{NAD}(\mathrm{P}) \mathrm{H}$ :quinine oxidoreductase 1 (NQO1). Nat Commun. 2015;6:7244.

48. Sun F, Zhang Y, Xu L, Li S, Chen X, Zhang L, Wu Y, Li J. Proteasome Inhibitor MG132 Enhances Cisplatin-Induced Apoptosis in Osteosarcoma Cells and Inhibits Tumor Growth. Oncol Res. 2018;26:655-64.

49. Dang L, Wen F, Yang Y, Liu D, Wu K, Qi Y, Li X, Zhao J, Zhu D, Zhang C, Zhao S. Proteasome inhibitor MG132 inhibits the proliferation and promotes the cisplatin-induced apoptosis of human esophageal squamous cell carcinoma cells. Int J Mol Med. 2014;33:1083-8.

50. Zhang L, Hu JJ, Gong F. MG132 inhibition of proteasome blocks apoptosis induced by severe DNA damage. Cell Cycle. 2011;10:3515-8.

51. Kang XH, Zhang JH, Zhang QQ, Cui YH, Wang Y, Kou WZ, Miao ZH, Lu P, Wang LF, Xu ZY, Cao F. Degradation of Mcl-1 through GSK-3beta Activation Regulates Apoptosis Induced by Bufalin in Non-Small Cell Lung Cancer H1975 Cells. Cell Physiol Biochem. 2017:41:2067-76.

\section{Publisher's Note}

Springer Nature remains neutral with regard to jurisdictional claims in published maps and institutional affiliations.

Ready to submit your research? Choose BMC and benefit from:

- fast, convenient online submission

- thorough peer review by experienced researchers in your field

- rapid publication on acceptance

- support for research data, including large and complex data types

- gold Open Access which fosters wider collaboration and increased citations

- maximum visibility for your research: over $100 \mathrm{M}$ website views per year

At BMC, research is always in progress.

Learn more biomedcentral.com/submissions 